\title{
LEVANTAMENTO EXPERIMENTAL GPR NO RIO TAQUARI, BACIA DO PANTANAL MATOGROSSENSE
}

\author{
Luiz Antonio Pereira de Souza, ${ }^{1}$ Jorge Luís Porsani, ${ }^{2}$ Osni Corrêa de Souza ${ }^{3}$ e Leandro Moutinho ${ }^{4}$ \\ Recebido em 15 jan., 2002 / Aceito em 29 out., 2003 \\ Received Jan. 15, 2002 / Accepted 0ct. 29, 2003
}

\begin{abstract}
An experimental investigation has been carried out using GPR - Ground Penetrating Radar method in Taquari River, located in Pantanal Matogrossense Basin Brazil, in order to understand the silting process on the river bottom and also to map the channels along the river, that is essential to local navigation. Many GPR profiles have been carried out in Lower Taquari river, near Santa Maria and Porto Santa Luzia farms. Preliminary interpretations were encouraging, allowing to located the channels of the river and the occurrence of very big sand deposits, showing the viability of GPR application to Taquari river sedimentation process studies. All data collected are now been analysed in order to understand the local dynamic sedimentation process, as part of a MSc. Dissertation in progress by an IAG-USP student. Results from these geophysical investigations are going to serve as guidance to multidisciplinary studies of Pantanal Program, by government of Mato Grosso do Sul State.
\end{abstract}

Keywords: GPR-Ground Penetrating Radar, Taquari River, Pantanal Wetland, silting up, River Channel.

\section{RESUMO}

Um levantamento experimental GPR (Ground Penetrating Radar) foi realizado no Rio Taquari, na Bacia do Pantanal Matrogrossense, Brasil, visando entender os processos de assoreamento na base do Rio e mapear os canais ao longo do Rio, que são essenciais para a navegação local. Muitos perfis GPR foram realizados no baixo Taquari, próximos à Fazenda Santa Maria e à Fazenda Porto Santa Luzia. Interpretações preliminares foram interessantes, permitindo localizar os canais do Rio e a ocorrência de grandes depósitos de areia, mostrando a viabilidade das aplicações do GPR em estudos de processos de sedimentação no Rio Taquari. Todos os dados adquiridos estão sendo analisados visando entender a dinâmica local nos processos de sedimentação, como parte de uma dissertação de mestrado em andamento por um estudante no IAG-USP. Os resultados deste levantamento geofísico servem com um guia para os estudos multidisciplinares do Programa Pantanal do governo do Estado do Mato Grosso do Sul.

Palavras-chave: GPR-Ground Penetrating Radar, Rio Taquari, Pantanal Matogrossense, assoreamento, canais do rio.

\footnotetext{
IPT - Laboratório de Geofísica Aplicada. Cidade Universitária. São Paulo - SP. Fax: 0xx11 3767 4767. Comercial: (11) 3767 4375. Email: laps@ipt.br

2 IAG-USP. Departamento de Geofísica. Rua do Matão 1226. Cidade Universitária. São Paulo - SP. CEP 05508-090. Fone: (11) 3091 4734. Fax: (11) 3091 5034. Email: porsani@iag.usp.br

3 Empresa Brasileira de Pesquisa Agropecuária (EMBRAPA / Gado de Corte) - Campo Grande/MS.

4 IAG-USP. Departamento de Geofísica. Rua do Matão 1226. Cidade Universitária. São Paulo - SP. CEP 05508-090. Fone: (11) 3091 4734. Fax: (11) 3091 5034. Email: Imout@bol.com.br
} 


\section{INTRODUÇÃO}

A Bacia Hidrográfica do Taquari, localizada na Região Centro Oeste do Brasil, é uma das principais drenagens formadoras da Alta Bacia do Rio Paraguai. Ela abrange a região de planaltos, também denominada Bacia do Alto Taquari e a Planície Pantaneira. A bacia tem como dreno principal o rio Taquari, cuja nascente localiza-se no estado do Mato Grosso, no município de Alto Taquari numa altitude de $860 \mathrm{~m}$ e, após percorrer $40 \mathrm{~km}$ nesse Estado, chega ao Estado de Mato Grosso do Sul.

A intensificação dos processos erosivos que ocorrem na Bacia do Alto Taquari, devido à ocupação agrícola intensificada a partir da década de 1970, tem sido apontada como o mais grave impacto ambiental e socioeconômico, devido ao assoreamento e inundações do rio Taquari na Planície Pantaneira, que é representada por um leque aluvial quaternário de aproximadamente $50.000 \mathrm{~km}^{2}$ ocupando cerca de $36 \%$ da área total do Pantanal brasileiro.

Um dos problemas da Bacia do Alto Taquari está relacionado com os processos erosivos, que têm como causas principais 0 desmatamento e o desconhecimento do comportamento evolutivo dos processos erosivos em escala local e regional. Os desmatamentos ocorrem devido à necessidade de plantio de subsistência da comunidade regional.

0 uso inadequado de ambientes vulneráveis à erosão intensifica os processos erosivos que evoluem na forma de voçorocas. Essas voçorocas podem ser consideradas como o mais sério problema erosivo da região. Como consequêencia imediata tem-se 0 aumento do volume de materiais transportados e depositados nas calhas dos rios e as alterações hidrológicas.

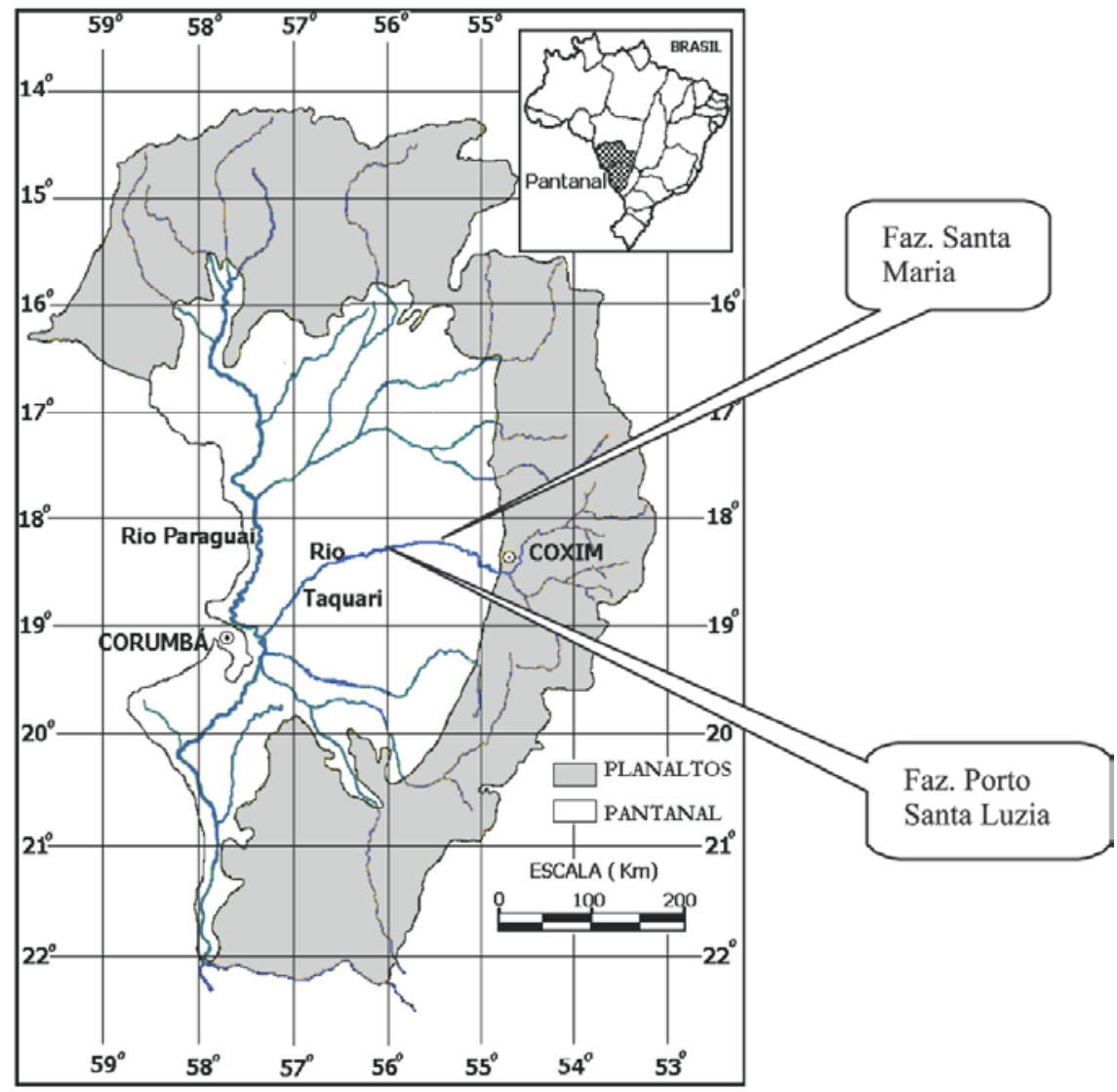

Figura 1 - Mapa de localização da área de estudos no estado de Mato Grosso do Sul (adaptado de SOUZA, 1998). Figure 1 - Location map of the studies area in the Mato Grosso do Sul State (after Souza, 1998). 
Na Planície Pantaneira, os principais problemas estão relacionados com o assoreamento generalizado do leito do rio Taquari, na região do leque aluvial, e com as alterações hidrológicas do rio, representadas pelas mudanças de curso e o regime de inundação, que por sua vez interfere na atividade pecuária, principal atividade socioeconômica do Pantanal.

As investigações geofísicas que utilizam o método GPR - Ground Penetrating Radar - têm como objetivos subsidiar os estudos multidisciplinares sobre a subcomponente Bacia do Taquarí, do Programa Pantanal, do Governo do estado de Mato Grosso do Sul (ARAÚJ0 et al., 1999).

\section{Aspectos Hidrológicos}

0 rio Taquari tem cerca de $1000 \mathrm{~km}$ de extensão e é um dos principais rios que cortam a bacia do pantanal matogrossense. Segundo Souza (1998), ao longo de seu curso, existem basicamente três características deposicionais em locais distintos: 0 primeiro local corresponde ao início dos processos deposicionais (médio Taquari), 0 segundo local corresponde ao ponto médio do rio entre o início da deposição e a planície pantaneira inundada (baixo Taquari) e o terceiro local corresponde à planície pantaneira constantemente inundada (baixo Taquari).

Na posição média entre o início da deposição e as áreas constantemente inundadas, o rio Taquari deixa de ser meandrante, mas ainda corre encaixado. Essas características ocorrem próximo à Fazenda Santa Maria, localizada às margens do rio Taquari e distante cerca de $160 \mathrm{~km}$ de Coxim. Neste trecho, o rio apresenta uma energia média, e os processos erosivos não são intensos, ocorrendo principalmente a deposição dos sedimentos menos grosseiros.

A planície pantaneira, constantemente inundada, é caracterizada pelo local onde o rio Taquari deixa o seu leito, ou seja, onde o rio deixa de correr encaixado, e passa a inundar as regiões vizinhas. Nas proximidades da Fazenda Porto Santa Luzia, distante cerca de 250km de Coxim, tem-se 0 início das inundações da planície. A partir daí, 0 nível da água do rio está acima do nível geral da planície de inundação. Os processos deposicionais ocorrem em função da capacidade e da competência do fluxo de água, sendo que os sedimentos mais grossos, tipo areia, permanecem em transporte no leito do rio e próximo às margens, e os sedimentos mais finos, tipo silte e argila, são transportados seguindo o fluxo das águas tanto no rio como na planície de inundação, podendo ser depositados em depressões afastadas do rio. Os freqüentes arrombamentos de margens, também conhecidos como "boca", no entanto, permitem alterações hidrológicas do Taquari. 0 redirecionamento parcial do fluxo do rio, através de canais distributivos, alteram o regime de inundação e, conseqüentemente, de deposição em vastas áreas da planície de inundação. Tais processos permitem uma contínua evolução geomórfica e geológica do leque aluvial do rio Taquari.

\section{Aquisição e Processamento dos Dados GPR}

Os trabalhos de campo foram realizados na região do baixo Taquari, planície do pantanal matogrossense. Participaram desse levantamento experimental os pesquisadores do IAG/USP, IPT e EmbrapaMS. Os dados foram adquiridos em duas áreas distintas: uma defronte à Fazenda Santa Maria e a outra próxima à Fazenda Porto Santa Luzia. A Figura 1 mostra 0 mapa de localização das áreas pesquisadas.

Os dados GPR foram adquiridos na forma de perfis de reflexão, longitudinais e transversais ao rio Taquari, utilizando-se antenas com frequêencias centrais em 50, 100 e 200MHz. Devido ao caráter experimental dessa pesquisa, vários parâmetros de campo foram testados, tais como, stacke o intervalo de tempo de aquisição.

Para a conversão dos perfis GPR obtidos em tempo (nanosegundos) para perfis em profundidade (metros), foi utilizada a velocidade de 0,05m/ns. 0 cálculo da velocidade de propagação da onda eletromagnética na água foi realizado através das medidas da profundidade do fundo do leito do rio e da leitura no radargrama do tempo duplo do sinal eletromagnético na água (PORSANI, 1999). Para este trabalho, a título de ilustração, foram selecionados dois perfis GPR, um adquirido na Fazenda Santa Luzia, e o outro na Fazenda Porto Santa Luzia (Figuras 2 e 3 ).

Os perfis GPR foram posicionados utilizando-se um equipamento de GPS diferencial modelo Pro-XL fabricado pela Trimble, pertencente ao IPT.

Os dados foram adquiridos com o equipamento Sueco Ramac/ GPR (Mala/ GeoScience) pertencente ao Departamento de Geofísica do IAG/USP. 0 equipamento foi adaptado numa embarcação alugada no local e as antenas foram colocadas em catamarãs, projetados e desenvolvidos pelos pesquisadores do Instituto de Pesquisas Tecnológicas de São Paulo. Alguns resultados de levantamentos GPR em lâmina d'água, podem ser encontrados em Sauck (1998); Aquino e outros (1998); Galli \& Souza (1999).

Os perfis de reflexão foram processados utilizando-se o software Gradix (Interpex). 0 processamento básico utilizado consistiu das seguintes etapas: a) aplicação de filtro de (correção do wow, que é um ruído de baixa frequiência); b) correção do tempo zero; c) aplicação de ganhos no tempo (filtro do tipo SEC - spherical exponential compensation, linear, 


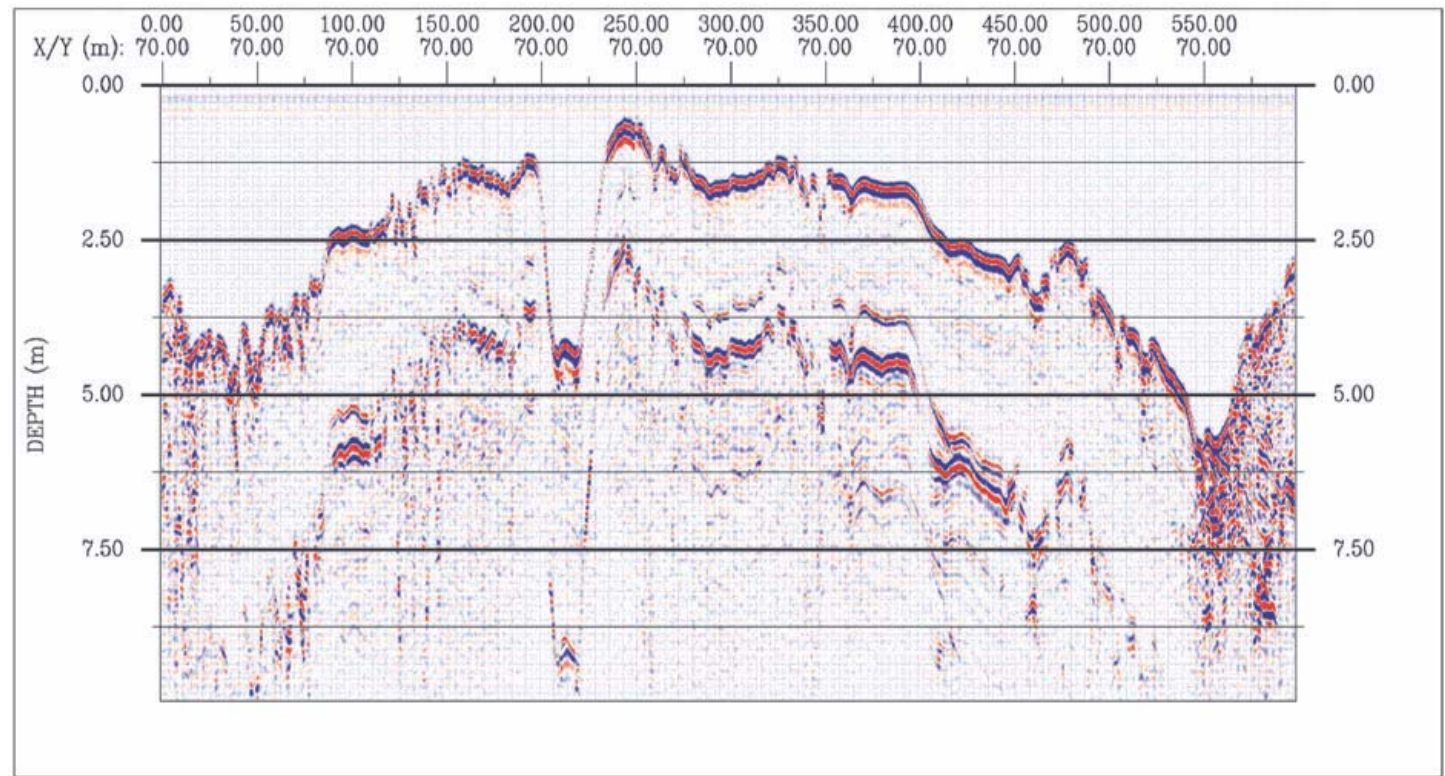

Figura 2 - Perfil de reflexão em profundidade obtido com as antenas de 200MHz, adquirido próximo à Fazenda Santa Maria. Figure 2 - Profile of reflection in depth obtained with 200 MHz antennas acquired near to Santa Maria Farm.

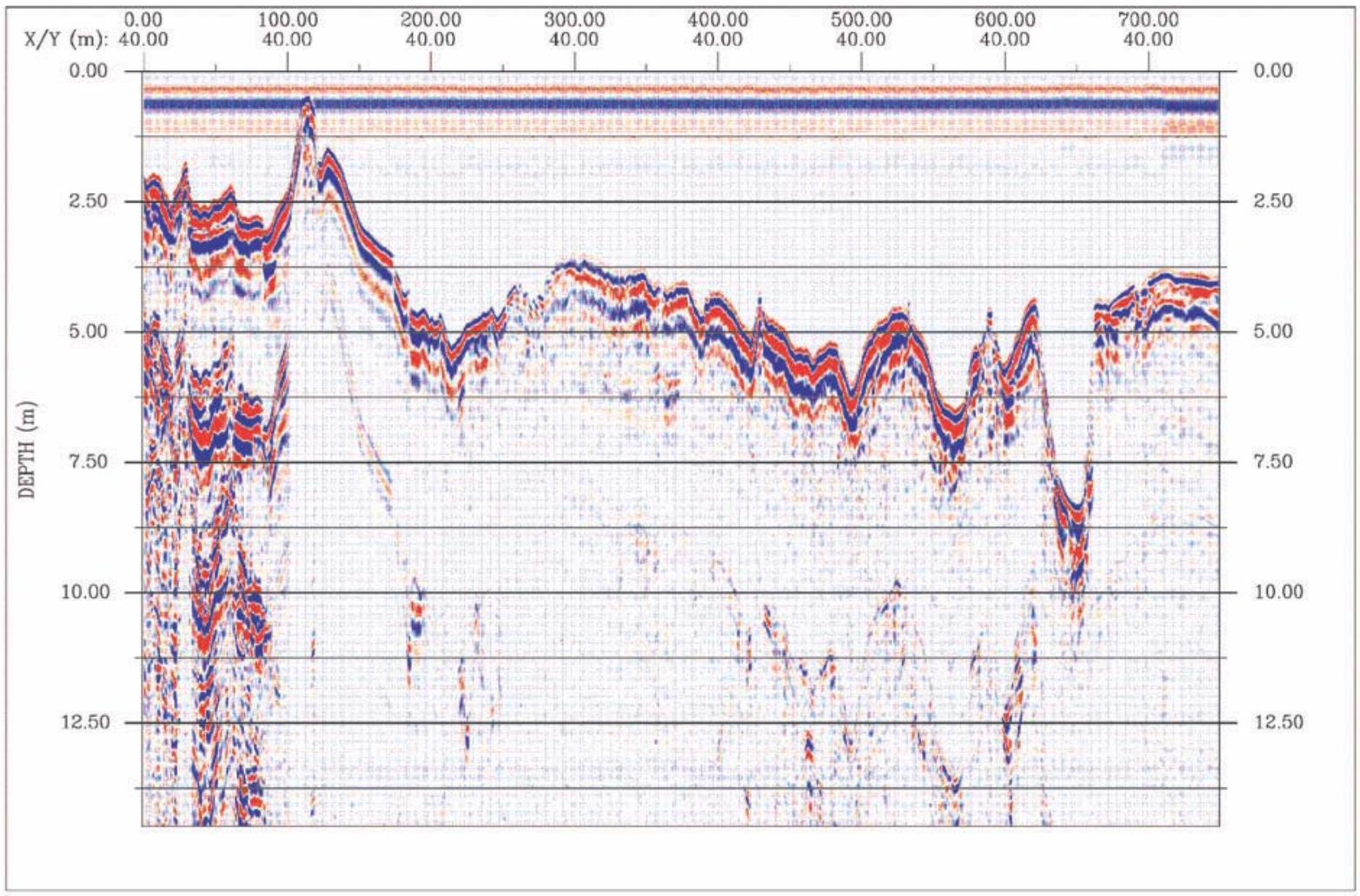

Figura 3 - Perfil de reflexão em profundidade obtido com as antenas de 100MHz, adquirido próximo à Fazenda Porto Santa Luzia. Figure 3 - Profile of reflection in depth obtained with $100 \mathrm{MHz}$ antennas acquired near to Santa Luzia Farm. 
constante e programado); d) aplicação de filtro do tipo passa banda e; e) aplicação de filtro espacial moving average (três traços).

\section{DISCUSSÃO PRELIMINAR DOS RESULTADOS}

Os perfis apresentados neste trabalho mostram que a lâmina d'água do rio Taquari (inferior a 10m nos trechos investigados), não foi obstáculo à propagação do sinal eletromagnético emitido pelas antenas de 50, 100 e 200MHz.

Os dois radargramas (Figuras 2 e 3 ) mostram imagens claras e detalhadas da superfície de fundo do rio. 0 primeiro refletor observado corresponde à topografia do fundo do rio; 0 segundo, mostrado de forma mais nítida no registro da Figura 2, corresponde à reflexão múltipla do primeiro refletor.

As reflexões rasas observadas nos perfis correspondem aos locais intensamente assoreados e referem-se aos bancos de areia que hoje ocupam grande parte do curso do rio Taquari. As partes mais profundas correspondem aos canais recentes do rio. Estes canais são de ocorrência bastante restrita, face ao intenso processo de assoreamento que assola a região nessas últimas três décadas.

Nota-se que a penetração do sinal de radar, além do leito do rio, com as três antenas utilizadas, limitou-se a $5-6 \mathrm{~m}$, sem alcançar um refletor que eventualmente pudesse ser correlacionado ao embasamento local. Todavia, a boa qualidade dos registros permite supor que 0 embasamento nos trechos estudados está além dos 5-6m observados. Outra alternativa para explicar a inexistência de um refletor bem definido que pudesse ser correlacionado a um embasamento local, seria a inexistência de constrastes, suficientemente expressivos, entre as propriedades físicas dos sedimentos saturados do leito do rio e do embasamento. Esse tema será investigado com mais detalhe nas próximas etapas de campo previstas ainda para este ano.

Por outro lado, os dados obtidos permitem analisar a geometria do refletor correspondente à superfície de fundo, que pode conter informaç̧eses importantes sobre os tipos de sedimentos (arenosos, argilosos etc.), contribuindo para o melhor entendimento do processo deposicional e, portanto, da dinâmica atual do rio Taquari. Este estudo sobre a arquitetura deposicional dos sedimentos está em desenvolvimento, e corresponde ao trabalho de dissertação de mestrado de um aluno do IAG-USP.

\section{Agradecimentos}

Ao Sr. Hélio Silva, proprietário da Fazenda Santa Maria, pela infra-estrutura e ao Sr. José Bispo ("Seu Zeca"), pela hospitalidade e apoio logístico. À Dra. Maria Ribeiro Araúio (Embrapa/MS), à Dra. Naomi Ussami (IAG/USP) e ao Dr. Francisco Yukio Hiodo (IAG/USP), pelas proveitosas discussões.

\section{REFERÊNCIAS}

AQUINO, W. F. et al. Aplicação de geo-radar em estudos de assoreamentos de reservatórios. In: Encontro Regional de Geotecnia e Meio Ambiente, 2.; Workshop de Geofísica Aplicada, 2., 1999, Rio Claro. Trabalho apresentado... Rio Claro: Fundação Unesp, 1998. CDROM.

ARAÚJO, M. R. et. al. Estudos multidisciplinares sobre o sub-componente Bacia do Taquari, do Programa Pantanal. Projeto de Pesquisa. Governo do Estado do Mato Grosso do Sul. Mato Grosso do Sul: Embrapa-MS/ IAG-USP/ IPT/UFMS, 1999.

GALLI, V. L.; SOUZA, L. A. P. GPR: exemplos de aplicação. In: International Congress of the Brazilian Geophysical Society, 6., 1999, Rio de Janeiro. Trabalhos apresentados... [Rio de Janeiro: s.n], 1999. CDROM.

PORSANI, J. L. Ground Penetrating Radar (GPR): Proposta metodológica de emprego em estudos geológico-geotécnicos nas regiōes de Rio Claro e Descalvado - SP. 1999. 145 p. Tese (Doutorado)-Instituto de Geociências e Ciências Exatas, Universidade Estadual Paulista, Rio Claro, 1999.

SAUCK, W. A. Use of a Bottom-towed GPR antenna for investigation of a Lake Michigan Contaminant Site. In: General Assembly of the European Geophysical Society, 20., 1998, Hamburg Annales Geophysicae..., [Hamburg: s.n.], 1998.

SOUZA, 0. C. Modern geomorphic processes along the Taquari River in the Pantanal: a model for development of a humid tropical aluvial fan. 1998. 135 p. Thesis (PhD), University of California, Santa Barbara, 1998. 


\section{NOTAS SOBRE OS AUTORES}

Luiz Antonio Pereira de Souza é geólogo do Laboratório de Geofíisca Aplicada do Instituto de Pesquisas Tecnológicas do Estado de São Paulo, desde 1981. Mestre em Oceanografia Geológica pelo Instituto Oceanográfico da USP. Doutorando em Oceanografia Geológica no Instituto Oceanográfico da USP. Atua na área de geofísica aplicada à investigação de áreas submersas, utilizando-se principalmente de métodos sísmicos de alta resolução (perfilagem sísmica contínua), sonar de varredura lateral, GPR e ecobatimetria.

Jorge Luís Porsani é geólogo pelo Instituto de Geociências da UFBA (1987). Mestrado em Geofísica pelo Núcleo de Pesquisas Geofísicas Aplicadas à Prospecção de Hidrocarbonetos da UFPA (1991). De 1991 a 1996, trabalhou como Geofísico no Centro de Pesquisas da PETROBRAS. Doutorado em Geociências e Meio Ambiente pelo Instituto de Geociências e Ciências Exatas da UNESP (1999). Desde 01/12/ 1998 é Docente do Departamento de Geofísica do IAG/USP, atuando com métodos geoelétricos aplicados à geologia, geotecnia, meio ambiente e arqueologia. Atualmente, é vice-coordenador do Programa de Pós-Graduação em Geofísica e Coordenador do Projeto de Instalação do Sítio Controlado de Geofísica Rasa do IAG (Fapesp 02/07509-1).

Osni Corrêa de Souza (in memorian) foi pesquisador científico do Centro Nacional de Pesquisa Gado de Corte-CNPGC/EMBRAPA. Engenheiro Agrônomo pela Escola de Agronomia e Veterinária de Jaboticabal-UNESP. M.Sc. em Ciências do Solo pela Texas A\&M University, College Station-USA. Ph.D. em Ciência e Manejo Ambiental pela University of California-Santa Bárbara-USA, com publicações nacionais e internacionais relevantes nas áreas de recursos naturais e meio ambiente nos ecossistemas Pantanal e Cerrado, na bacia hidrográfica do TAQUARI. Professor do Curso de Pós-Graduação em Meio Ambiente da Universidade para o Desenvolvimento do Estado e Região do Pantanal-UNIDERP, área de Hidrologia. Membro titular de vários comitês técnico-cientíicos relacionados às áreas de geohidrossedimentologia e meio ambiente no Estado do Mato Grosso do Sul e, membro associado a American Geophysical Union-USA.

Leandro Moutinho é bacharel em Geofísica pelo Instituto de Astronomia, Geofísica e de Ciências Atmosféricas da Universidade de São Paulo (IAG - USP) em 2000. Obteve o tíitulo de Mestre em Geofísica pelo IAG - USP em 2003, desenvolvendo a pesquisa intitulada: "Processamento de dados GPR adquiridos no Rio Taquari - Pantanal Matogrossense, com ênfase em deconvolução". Atualmente trabalha como Geofísico na área de aquisiçã̃o, processamento e interpretação de dados geofísicos junto à empresa AeroGeoPhysica Latinoamerica (AGP - LA). 